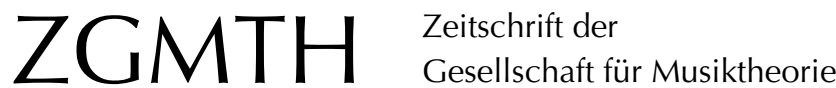

Sprenger, Sebastian (2003/05): „Winkelschiefe Satzkunst«. Zu einigen Quint- und Oktavparallelen im Werk Gustav Mahlers. ZGMTH 1-2/1/1, 85-105.

https://doi.org/10.31751/485

(C) 2003/05 Sebastian Sprenger

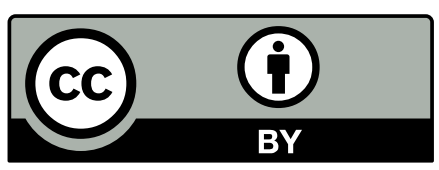

Dieser Text erscheint im Open Access und ist lizenziert unter einer Creative Commons Namensnennung 4.0 International Lizenz.

This is an open access article licensed under a Creative Commons Attribution 4.0 International License.

veröffentlicht / first published: 01/01/2003

zuletzt geändert / last updated: 15/09/2009 


\title{
»Winkelschiefe Satzkunst« \\ Zu einigen Quint- und Oktavparallelen im Werk Gustav Mahlers
}

\author{
Sebastian Sprenger
}

In den Liedern und Symphonien Gustav Mahlers begegnen offene Quint- und Oktavparallelen zu häufig, als daß sie als marginale Erscheinungen anzusprechen wären. Der vorliegende Aufsatz skizziert eine Typologie der kompositorischen Kontexte, in die diese nach herkömmlichem Verständnis fehlerhaften Fortschreitungen eingebettet sind. Dies führt zu einer Polarisierung: Auf der einen Seite finden sich Satztechniken, die zum tradierten Tonsatz gleichsam a priori querstehen (etwa Parallelverschiebung grundstelliger Dreiklänge oder heterophone Bildungen) und sich somit am besten aus ihrer Eigengesetzlichkeit beschreiben lassen; dem stehen traditionsnähere Strukturen (choralartige oder kontrapunktische Passagen) gegenüber, innerhalb derer den genannten Parallelen eher eine Funktion als - wie auch immer motivierte - Abweichung, als Negation einer Norm zukommt. Es ergibt sich die eigentümliche Ambivalenz, daß möglicherweise gerade der von Mahler selbst beklagte Mangel an kontrapunktischer Routine die Voraussetzung für satztechnische Optionen bot, die sich aus heutiger Sicht durchaus modern, zumindest charakteristisch für Mahlers Personalstil, ausnehmen.

In ihren Erinnerungen an Gustav Mahler berichtet Natalie Bauer-Lechner unter dem Eintrag des 21. Oktobers 1901 folgende Begebenheit:

Mahler, der nach dem heißen Tagewerk um 10 Uhr von einer Probe heimkam, arbeitete nach dem Abendmahl, wie er pflegte, an der Korrektur seiner Vierten, wobei er unversehens auf eine falsche Fortschreitung stieß. Er war empört, daß ihm das passiert sei. , Die hat mich durch ihre Ordinärheit bei jedem Hören schon gestört, ohne daß ich doch drauf gekommen wäre. Wenn einem so etwas in die Partitur kommt, ist es, wie wenn jemand von hohem Adel in seinem Stammbaum plötzlich einen - Sauhirten entdeckter (Bauer-Lechner 1984, 199).

Bauer-Lechner fährt fort:

Er [Mahler] und Walter ${ }^{1}$ sprachen davon, wie häufig solche verdeckten Oktaven bei anderen Meistern sich fänden: bei Bach, Schumann usw. 'Nur bei Beethoven`, sagte Mahler, sbin ich nie darauf gekommen. Diese haben aber die Entschuldigung, daß sie eine große Menge geschrieben haben. Wenn man aber alle Jahre nur ein Werk vollbringt, darf das nicht geschehen!^ (ebd.)

1 Gemeint ist der Dirigent und langjährige Freund Mahlers Bruno Walter. 
Wer mit dem Werk Gustav Mahlers einigermaßen vertraut ist, für den enthält dieser Bericht - will man ihm Glauben schenken - Erstaunliches: Mahler reagiert empört auf die Entdeckung einer verdeckten Oktavparallele, einer im strengen Satz vergleichsweise harmlosen Sünde, in einer eigenen Partitur. Wollte man jedoch Mahlers Partitur auf Verstöße gegen den strengen Satz hin durchforsten, so stieße man nicht selten auf weit Gravierenderes: offene Quintparallelen in großer Zahl, seltener sogar offene Oktavparallelen, die sich nicht auf die konsequente Oktavierung eines Instrumentalparts durch einen zweiten (etwa der Violoncellostimme durch die Partie der Kontrabässe) zurückführen lassen. Sollte man all diese Stellen nur als satztechnische Flüchtigkeitsfehler bewerten, die Mahler bei sgründlicherer Arbeit vermieden hätte?

Gut hundert Jahre später, da so unterschiedliche Komponisten wie Claude Debussy - immerhin ein Zeitgenosse Mahlers - oder Carl Orff längst offene Quint- und Oktavparallelen im Rahmen ausgedehnter Mixturbildungen als stilbildende Gestaltungsmittel erkannt und weidlich ausgenutzt haben, ist man wohl eher geneigt, derlei Regelwidrigkeiten einen eigenen >Reiz zuzugestehen, vielleicht sogar in ihnen ein konstitutives Element für Mahlers Satztechnik zu sehen. So schreibt etwa Claus-Steffen Mahnkopf in seinem Aufsatz Mahlers Gnosis: »Mahlers sunorthodoxe`, ja winkelschiefe Satzkunst ist nicht einmal in Ansätzen untersucht; allein über die virtuose Handhabung von Quintparallelen oder die Sphäre neapolitanischer Phänomene ließen sich umfangreiche Spezialstudien anfertigen « (Mahnkopf 1996, 36f.). In der vorliegenden Arbeit, wahrhaftig keiner umfangreichen Spezialstudie, möchte ich immerhin den Versuch machen, darzustellen, innerhalb welcher kompositorischer Texturen Mahler offene Quint- und Oktavparallelen einsetzt, wobei ich, zu des Lesers und meiner eigenen Bequemlichkeit, auf die Präzisierung soffen im weiteren Verlauf des Textes verzichte - wo von Quint- resp. Oktavparallelen die Rede ist, sind damit also stets `offene` gemeint. Eine Darstellung verdeckter Parallelen geriete wohl gänzlich ins Uferlose; aber auch innerhalb der Grenzen meines Themas beanspruche ich keine Vollständigkeit der von mir skizzierten Systematik, erst recht nicht der zitierten Passagen aus Mahlers Partituren.

Integriert in eine Parallelverschiebung grundstelliger Dreiklänge begegnen Quintparallelen in Mahlers frühem Lied Ablösung im Sommer (Bsp. 1 und 2). Diese WunderhornVertonung bildet bekanntlich den Ausgangspunkt für das Scherzo der 3. Symphonie und wird dort zu Beginn nahezu vollständig - bis auf die letzten fünf Takte - zitiert. Das Prinzip der in Stufen fallenden Dreiklangsbewegung findet sich wieder in dem späteren Wunderhorn-Lied Lob des hohen Verstandes (Bsp. 3). Auch der Klaviersatz weist hier auffällige Ähnlichkeit auf (Austerzung und Spiel mit den oberen Wechselnoten in der rechten Hand wie in Bsp. 1; Oktavsprünge im Baß wie in Bsp. 1 und 2). In den folgenden beiden Beispielen (Bsp. 4 und 5) aus der 4. Symphonie ist der Klangwert der reinen Quinte besonders hervorgehoben. Die Terz als Füllton ist hier jeweils einer anderen kontrapunktischen Schicht des Satzes zugeordnet (in Bsp. 4 erscheint sie integriert in die Sechzehntelgirlanden der hohen Holzbläser und Streicher, in Bsp. 5 in die - durch Glissandi überbrückten - Dezimensprünge der Violoncelli). Interessant an Bsp. 5 ist zudem das Alternieren von reinen Quintklängen ohne Terzauffüllung (T. 41 und 43) mit vollständigen Dreiklängen (T. 42 und 44) sowie der durch die unterschiedliche Instrumentierung (T. 41 und 43: Violen und Violoncelli pizz.; T. 42 und 44: II. Violinen und Violen mit dem Bo- 


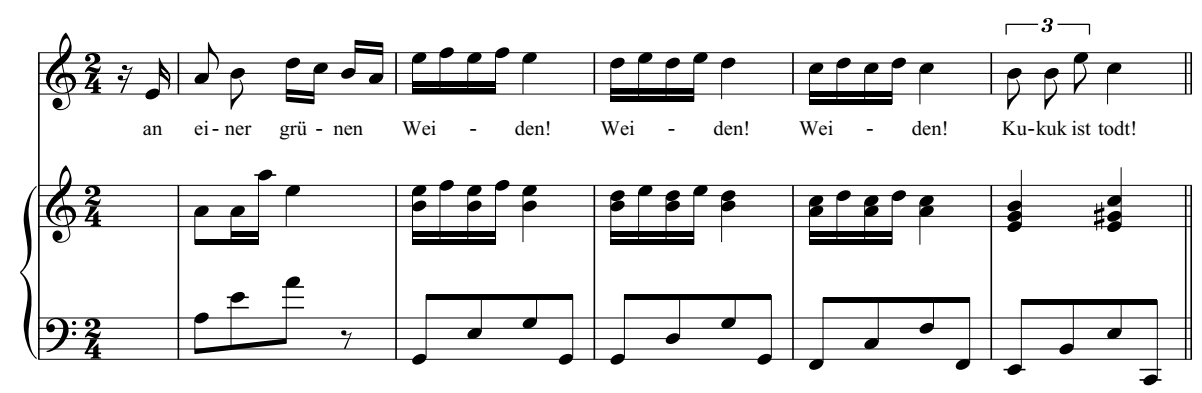

Beispiel 1: Gustav Mahler: Ablösung im Sommer (Fassung für tiefe Stimme), T. 6-10

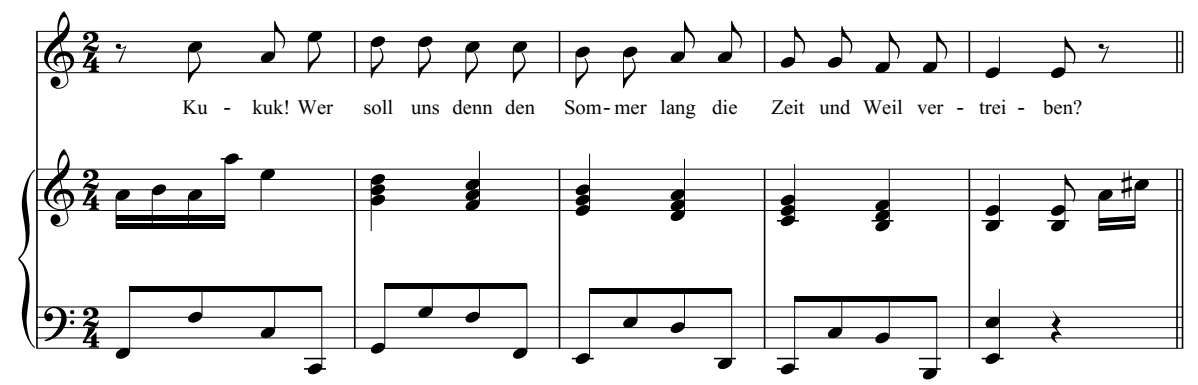

Beispiel 2: Gustav Mahler: Ablösung im Sommer (Fassung für tiefe Stimme), T. 27-31

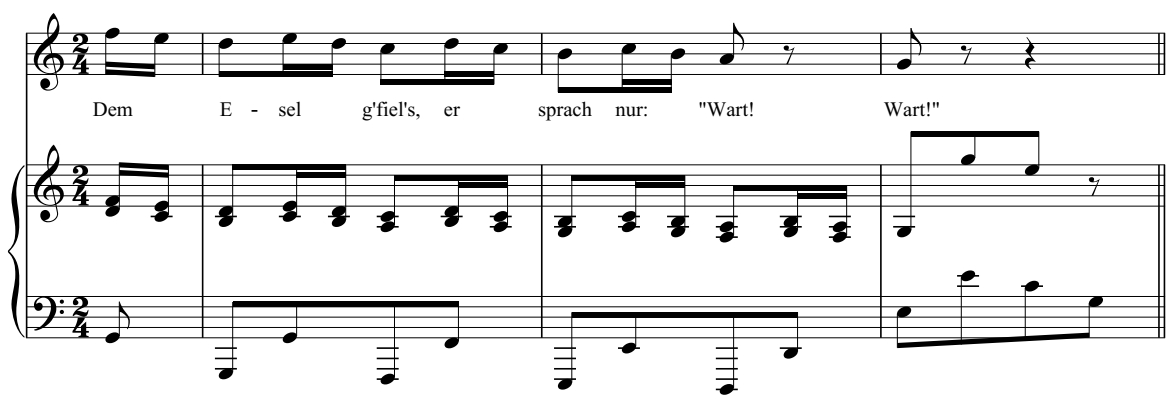

Beispiel 3: Gustav Mahler: Lob des hohen Verstandes (Fassung für tiefe Stimme), T. 94-96

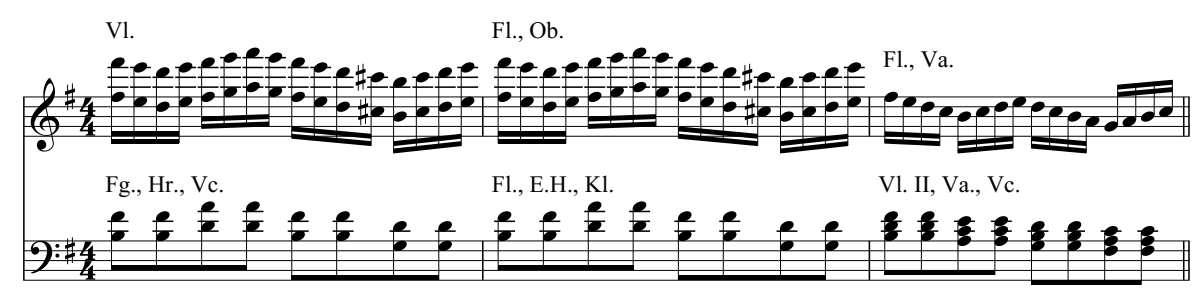

Beispiel 4: Gustav Mahler: 4. Symphonie, IV. Satz T. 45-47 


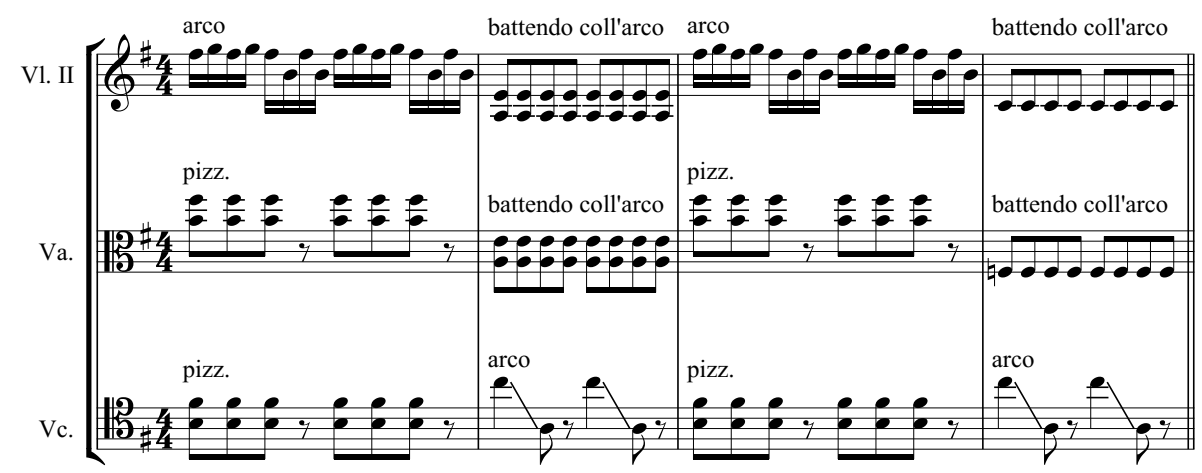

Beispiel 5: Gustav Mahler: 4. Symphonie, IV. Satz T. 41-44

gen geschlagen in durchgehenden Achteln) erzeugte 'Stereo -Effekt. Die eigenartige Mischung aus kindlich-verspielter Naivität und unverhohlener Brutalität (»Sanct Lucas den Ochsen tät schlachten / ohn' einig's Bedenken und Achten«), die der Text - wiederum ein Gedicht aus Des Knaben Wunderhorn - beschreibt, findet eine sinnvolle Entsprechung in
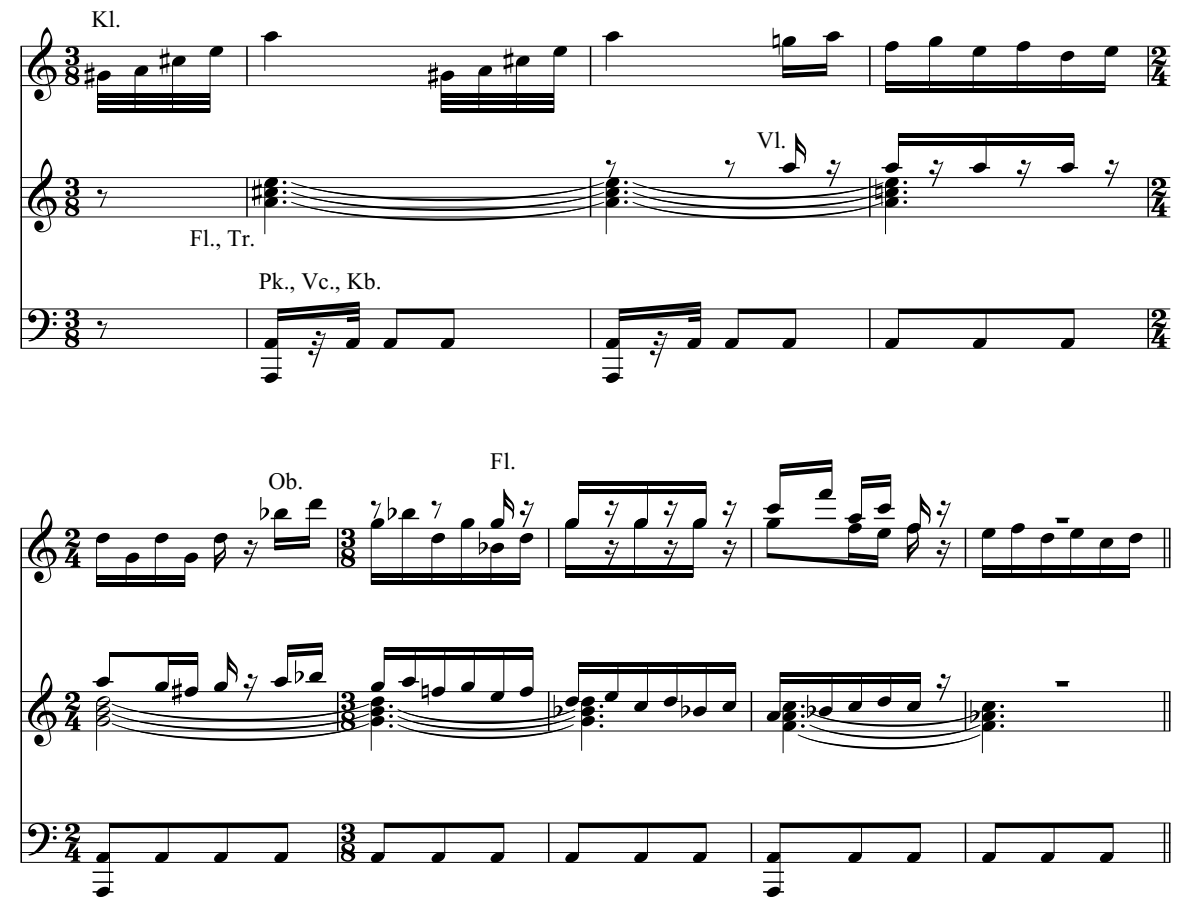

Beispiel 6: Gustav Mahler: 6. Symphonie, II. Satz (Scherzo), T. 419-426 


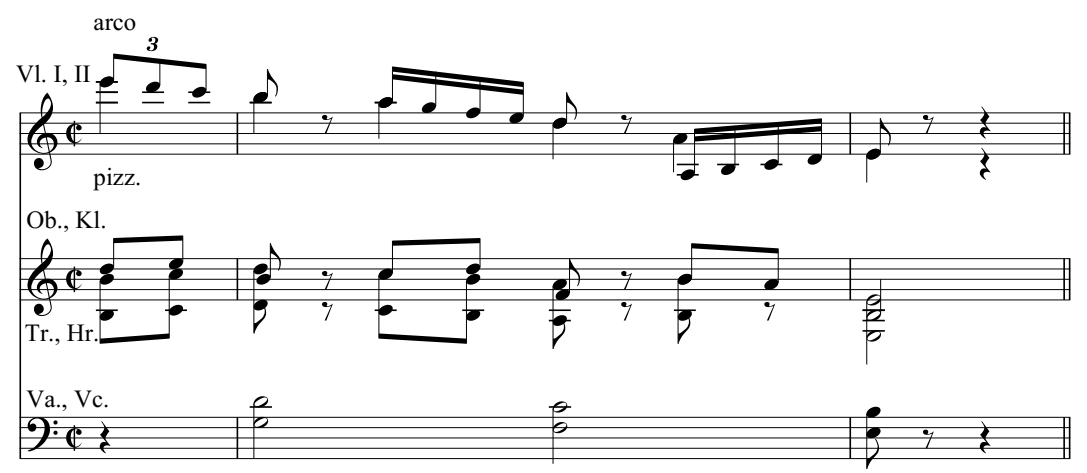

Beispiel 7: Gustav Mahler: 9. Symphonie, II. Satz (Rondo-Burleske), T. 21-22

der zugleich infantilen und rohen Satztechnik (wobei nicht eigens betont werden muß, daß eben dieses >Rohe und >Infantile $<$ mit großer Raffinesse in Szene gesetzt ist).

Im späteren Werk Mahlers, also etwa ab der Gruppe der mittleren Symphonien (5-7), treten derlei elementare Klangwirkungen eher in den Hintergrund. Gleichwohl lassen sich die nachfolgenden Takte aus dem Scherzo der 6. Symphonie (Bsp. 6) durchaus als Nachhall der oben beschriebenen Dreiklangsverschiebungen deuten, nun allerdings verschränkt mit der Abdunklung des Durdreiklanges zum gleichnamigen Molldreiklang, der in diesem Werk eine auffällige Rolle zugewiesen ist. Nebenbei bemerkt zeigt das harmonische Geschehen in Bsp. 6, verglichen mit dem der ersten vier Notenbeispiele, eine leichte Verschiebung von diatonischen zu distanziellen Tonordnungen: In den früheren Beispielen wird die Aufeinanderfolge von Dur- und Molldreiklängen durch den Tonvorrat der jeweiligen Tonart geregelt, sie - und damit auch der Wechsel von Halb- und Ganztonschritten in den einzelnen Stimmen - gehorcht keiner immanenten Logik. In Bsp. 6 hingegen stehen die ganztönig fallenden Außenstimmen der halbtönig fallenden Mittelstimme gegenüber - freilich auch hier nur eine höchst temporäre Logik, die sogleich wieder verlassen wird. Als vorbeihuschende Reminiszenz an die zitierten Passagen aus der 4. Symphonie erscheinen die folgenden anderthalb Takte aus der RondoBurleske der 9. Symphonie (Bsp. 7) mit ihren fallenden, gleichsam ınackten Quinten in den Violen und Violoncelli. Die übrigen Stimmen machen jedoch - wie übrigens auch schon bei Bsp. 6 - deutlich, daß der musikalische Satz, trotz vereinzelter Mixturbildungen, viel konsequenter von kontrapunktischem Denken bestimmt ist.

Das folgende Beispiel (Bsp. 8) aus Es sungen drei Engel einen süßen Gesang² mag überleiten zu einem zweiten Typus musikalischer Texturen, der sich - zugegebenermaßen etwas pauschal - unter dem Stichwort `Choral-Idiom` zusammenfassen läßt. So un-

2 Diese Wunderhorn-Vertonung findet sich in leicht erweiterter Form im fünften Satz der 3. Symphonie wieder, ähnlich wie Urlicht in der 2. Symphonie. Das himmlische Leben, welches das Finale der 4. Symphonie bildet, wurde von Mahler nicht in die Sammlung der 14 Lieder aus Des Knaben Wunderhorn aufgenommen. 


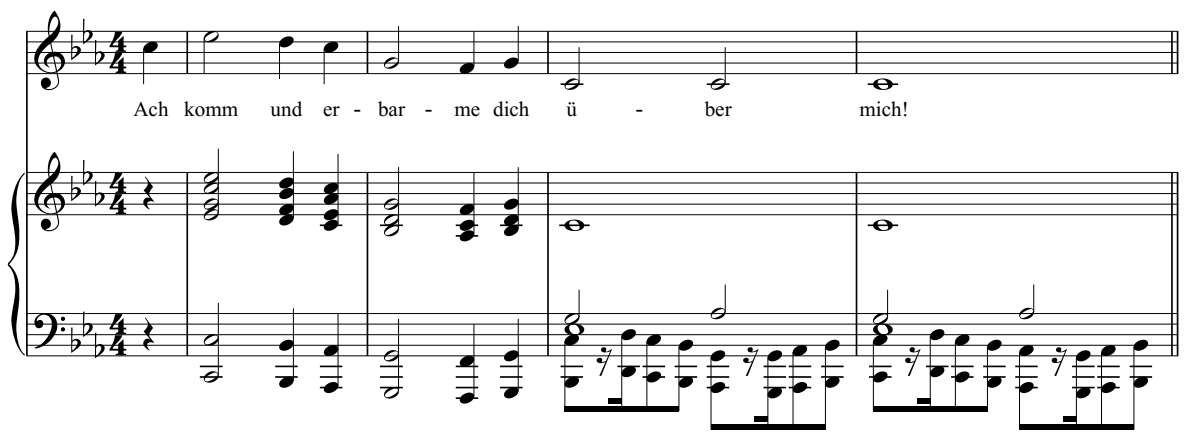

Beispiel 8: Gustav Mahler: Es sungen drei Engel einen süßen Gesang (Fassung für tiefe Stimme), T. 58-61

willkürlich sich die Choral-Assoziation häufig einstellt, so mühsam erscheint der Versuch, satztechnisch konkret zu benennen, was nun im jeweils einzelnen Fall ausschlaggebend für die Zuordnung zu diesem Typus ist: Ist es der homophone (homorhythmische) Satz? Eine diatonische Oberstimmenmelodie? Eine an die Zeilenform angelehnte musikalische Syntax? Sind es gar vergleichsweise ıäußerliche` Kriterien wie ein getragenes Tempo oder die Instrumentierung (Blechbläser-Satz)? - Im Zusammenhang der vorliegenden Arbeit ist eine stringente, eindeutige Definition des Phänomens `Choral-Idiom`nicht erstrebt. Gemeinsames Moment der nachfolgenden Beispiele ist in der Tat eine weitgehend gleiche Rhythmisierung der Einzelstimmen sowie ein figurationsarmer bis -freier Satz. Zurück zu Bsp. 8: Eine solch unverschleierte Anwendung des Mixturprinzips über sieben Akkorde hinweg stellt in Mahlers Schaffen einen Extremfall dar (zusammen mit den in Bsp. 2 zitierten Takten aus Ablösung im Sommer, die gleichfalls ihren Weg in die 3. Symphonie gefunden haben) - unweigerlich fühlt man sich erinnert an eine Passage aus Thomas Manns Doktor Faustus, nämlich den Bericht über einen Vortrag Wendell Kretzschmars (Adrian Leverkühns musikalischem Mentor) über »Das Elementare in der Musikı oder >Die Musik und das Elementare` oder `Die musikalischen Elemente noch etwas anders « (Mann 1990, 87). Dort heißt es vom Vortragenden: »Und er gedachte Anton Bruckners, der es geliebt habe, sich an der Orgel oder am Klavier durch das ein-

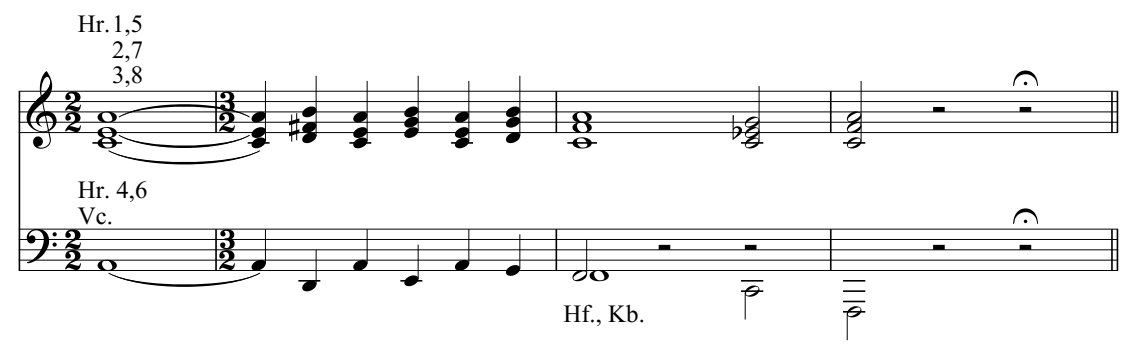

Beispiel 9: Gustav Mahler: 3. Symphonie, IV. Satz, T. 14-17 
fache Aneinanderreihen von Dreiklängen zu erquicken. ১Gibt es denn etwas Innigeres, Herrlicheres`, habe er gerufen, sals eine solche Folge bloßer Dreiklänge? Ist es nicht wie ein reinigendes Seelenbad?« (ebd., 88) Alle Oberstimmen gehen hier in Dreiklangsmixturen zum Baß parallel, wobei die Melodiestimme von der anfänglichen Dezimenmixtur beim Übergang zu T. 59 in die Oktavmixtur springt. In einer derartigen Passage sind die traditionellen Stimmführungsregeln gänzlich außer Kraft gesetzt, was in dieser Kon-

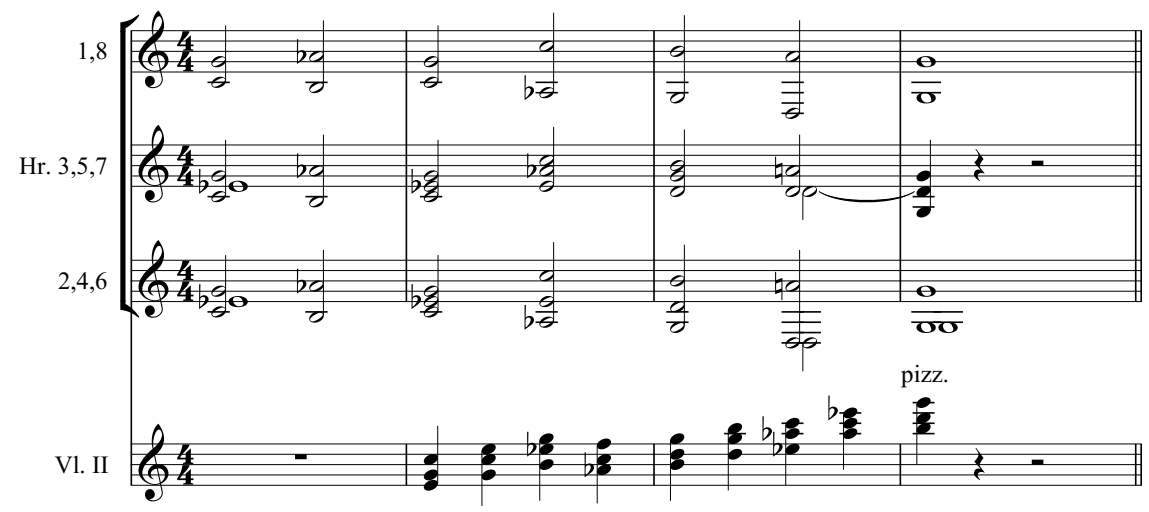

Beispiel 10: Gustav Mahler: 6. Symphonie, I. Satz, T. 210-213
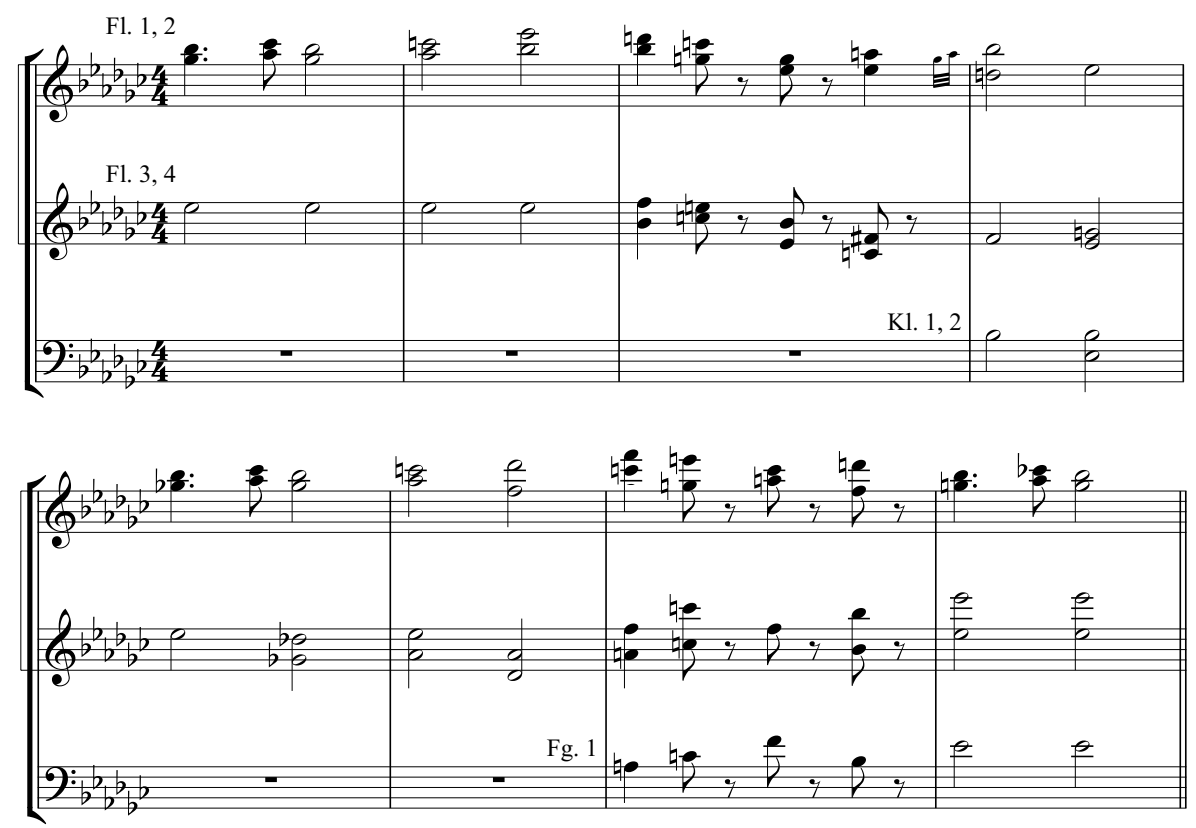

Beispiel 11: Gustav Mahler: 8. Symphonie, II. Satz, T. 147-154 


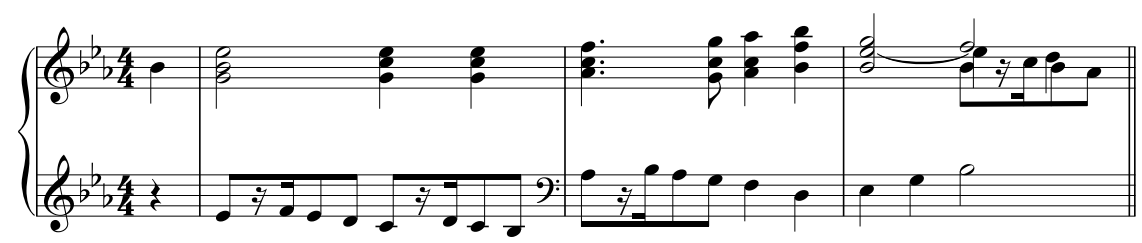

Beispiel 12: Gustav Mahler: Es sungen drei Engel einen süßen Gesang (Fassung für tiefe Stimme), T. 1-3

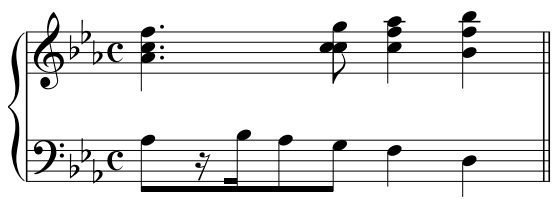

Beispiel 13: Erster 'Korrekturvorschlag` zum zweiten Takt von Bsp. 12

sequenz, wie gesagt, bei Mahler sehr selten zu beobachten ist. Häufiger innerhalb des Choral-Idioms begegnen einzelne satztechnische sFlecken`, punktuelle Verstöße gegen die tradierten Regeln. Dies geschieht entweder in der Form, daß ein kompletter Akkord aufwärts oder abwärts transponiert wird (meist um ein Sekundintervall; [Bsp. 9-11], T. 151 auf T. 152) - mit daraus resultierenden Quint- und Oktavparallelen - oder in einer Mischung korrekter und verbotener 'Fortschreitungen (Bsp. 11, T. 152: Hier wird nur das Quintfundament der beiden Stimmen verschoben). ${ }^{3}$

Ein extremes Beispiel an Verballhornung tonsetzerischen Handwerks findet sich schließlich gleich zu Beginn des schon zitierten Es sungen drei Engel einen süßen Gesang. Innerhalb eines ansonsten durchaus konventionell gehaltenen vierstimmigen Satzes verdoppelt im zweiten Takt die Unterstimme der rechten Hand drei Töne der Oberstimme in der Unteroktave (Bsp. 12). Zudem ergeben sich Oktaven zum Baß beim Übergang zum vierten Achtel. Erstaunlich sind diese Parallelen insofern, als sie einerseits weder direkt beim Hören auffallen - somit schwerlich als bewußt intendierter klanglicher ,Effekt gedeutet werden können -, andererseits ebensowenig als `Resultat `einer akkordischen Parallelverschiebung verständlich sind. Ohne Schwierigkeiten, d. h. vor allem ohne Eingriffe in die harmonische Substanz, ließe sich diese Stelle im traditionellen Sinne sberichtigen` (Bsp. 13). Nähme man an der klanglichen `Dünnheit` des vierten Achtels - bedingt durch die momentane Dreistimmigkeit sowie den kahlen Quart-Quint-Klang - Anstoß, wären auch Sextparallelen zwischen den Außenstimmen der rechten Hand denkbar (Bsp. 14). Interessant wäre es, experimentell zu erfahren, ob derartige Abwandlungen, die für die meisten HörerInnen auf bewußter Ebene sicher unbemerkt blieben, unterschwellig doch einen veränderten Höreindruck oder Charakter der besagten Passage mit sich brächten. Durch die Oktavparallelen erhält der melodische Aufschwung im zweiten

3 Genaugenommen erzeugt natürlich auch die Parallelverschiebung grundstelliger Dreiklänge nicht nur verbotene Fortschreitungen, sondern - je nach Lage - >legitime` Terz- (resp. Dezimen-) oder Sextparallelen. 


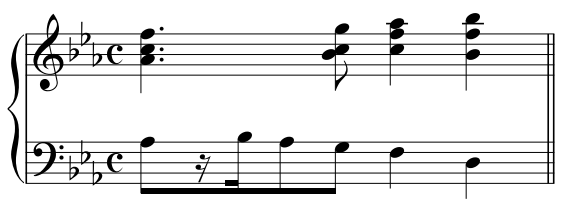

Beispiel 14: Zweiter $/$ Korrekturvorschlag, zum zweiten Takt von Bsp. 12

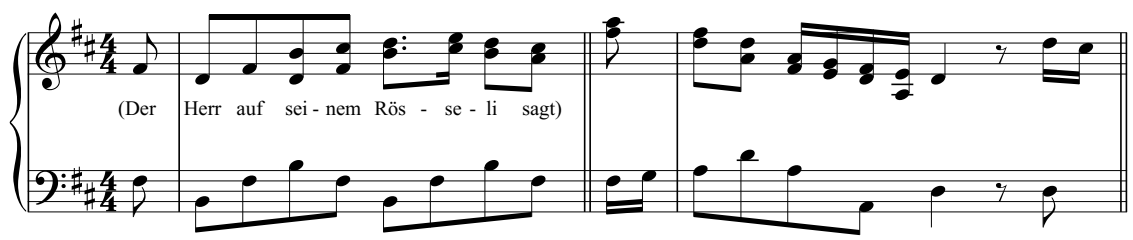

Beispiel 15: Gustav Mahler: Um schlimme Kinder artig zu machen (Fassung für tiefe Stimme), T. 13/19

Takt - nach der Stagnation im ersten - jedenfalls etwas `Forciertes`, `Angestrengtes`, letztlich eine Intensität, die den beiden `Korrekturvorschlägen - zumindest in meiner subjektiven Wahrnehmung - fehlt.

Ein weiterer Typus von Parallelen ergibt sich aus quasi zufälligen Koinzidenzen einer frei geführten Stimme und einer Ostinato-Figur in einer zweiten Stimme. Meist handelt es sich bei derlei Ostinati - wie in Bsp. 15, Bsp. 17 und Bsp. 19 - um I-V-Pendel. Die traditionelle Begründung für das Verbot von Oktav- und Quintparallelen - der Verlust der Selbständigkeit der einzelnen Stimmen - erscheint dabei wie auf den Kopf gestellt: Gerade weil die einzelnen Stimmen oder Schichten, vom gemeinsamen harmonischen Bezugsrahmen abgesehen, völlig selbständig, ja untereinander geradezu beziehungslos verlaufen, sind parallele Fortschreitungen (meist handelt es sich hier um Oktavparallelen) zwischen ihnen möglich, ohne in diesem Kontext besonders aufzufallen: Sie geschehen einfach (Bsp. 15). Ob man die sequenzierten Sekundpendel der Mittelstimmen (Bsp. 16) als Ostinato-Figur deuten soll, bleibt fraglich; mit gleichem Recht ließe sich hier von einer leicht ornamentierten doppelten Mixtur zur Oberstimme sprechen. Oktavparallelen ergeben sich (Bsp. 17) nicht nur im Verhältnis der am Kanon beteiligten Stimmen bzw. des Oboen-Kontrapunktes zum I-V-Ostinato der Pauken und tiefen Streicher. Sie lassen sich auch zwischen der Oboen- und den Kanonstimmen finden. Hier dürfte es vor allem das charakteristische Timbre der Oboe sein, das sich vom sonstigen Klanggeschehen deutlich abhebt, welches einer Verschmelzung der unterschiedlichen Schichten entgegenwirkt.

Eine diminuierte Variation zu Bsp. 16 läßt sich mit (sehr) viel Phantasie im Beginn der 4. Symphonie (Bsp. 18) erblicken: Im zweiten Takt ergeben sich aus der Kombination des Obersekundpendels der dritten und vierten Flöte mit der sich langsam abwärts schlängelnden Sechzehntel-Linie der ersten beiden Klarinetten Oktavparallelen. Dies geschieht freilich in einer Geschwindigkeit, die den Nachvollzug beim Hören, wenn man nicht ohnehin darum weiß, nahezu unmöglich macht. Ein reizvolles Beispiel von Gegenquintparallelen zwischen 


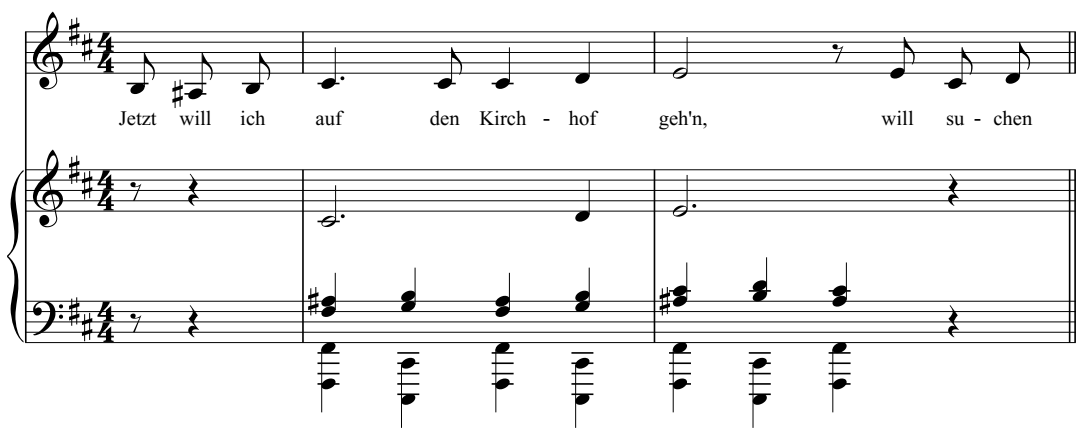

Beispiel 16: Gustav Mahler: Nicht wiedersehen! (Fassung für tiefe Stimme), T. 41-42

Fl. 1-4 E.H, Kl., Fg., Vl. 1 (pizz.)

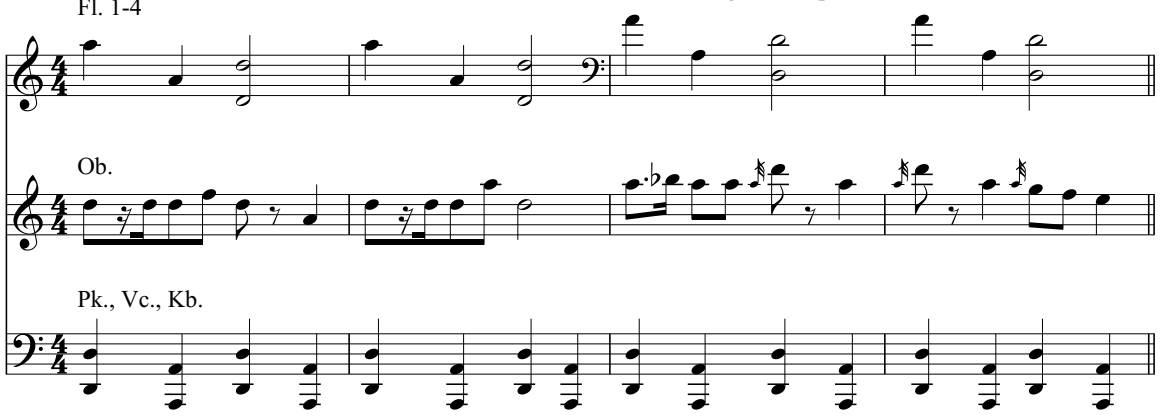

Beispiel 17: Gustav Mahler: 1. Symphonie, III. Satz, T. 29-32

Fl. 1,2

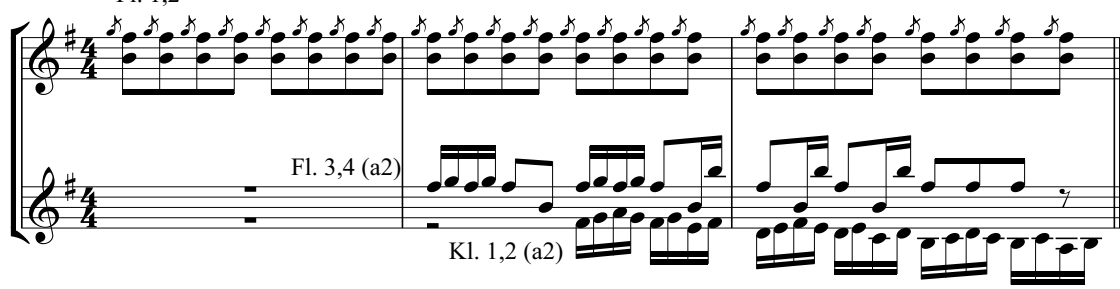

Beispiel 18: Gustav Mahler: 4. Symphonie, I. Satz, T. 1-3

Singstimme und linker Hand des Klavierparts (wiederum im I-V-Pendel) findet sich in Wo die schönen Trompeten blasen (Bsp. 19). Die Linie der Gesangspartie wird in der Begleitung gleichsam aufgeteilt unter den zwei - vereinzelt drei - Stimmen der rechten Hand. 


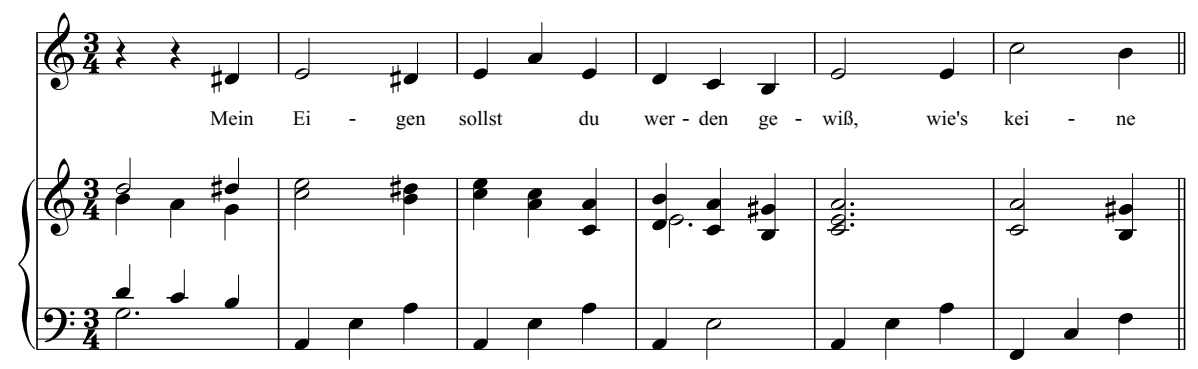

Beispiel 19: Gustav Mahler: Wo die schönen Trompeten blasen (Fassung für tiefe Stimme), T. $145-150$

Ein weiterer Satztypus, der die Bildung von Oktav- (und Einklangs-)Parallelen naturgemäß außerordentlich begünstigt, läßt sich unter dem - in der musikwissenschaftlichen Literatur allerdings umstrittenen und nicht einheitlich definierten - Terminus ১Heterophonie $\varsigma^{4}$ subsumieren. Gemeinhin wird damit eine Satzstruktur zwischen Einstimmigkeit und sechter Mehrstimmigkeit bezeichnet: Ein Instrument, eine Stimme umschreibt oder ornamentiert die Melodieführung eines/einer zweiten, bzw. beide umspielen auf je eigene Weise einen gemeinsamen >Melodiekern Begriff meist zur Charakterisierung verschiedener fernöstlicher Musizierpraktiken gebraucht. In der westlichen Kunstmusik ist dieser Satztypus bis zum 19. Jahrhundert selten anzutreffen; Pierre Boulez weist in seiner Schrift Musikdenken heute (Boulez 1963, 103) auf ein Beispiel aus dem Variationssatz aus Beethovens 9. Symphonie hin (Bsp. 20).

$\mathrm{Ob}$ und in welchem Maße Mahler bei der Komposition des Lieds von der Erde auf eigene Höreindrücke fernöstlicher und speziell chinesischer Musik zurückgreifen konnte, ist nicht eindeutig belegt; Constantin Floros vermutet die eigentliche Inspirationsquelle für Mahlers musikalische Chinoiserien in Giacomo Puccinis Oper Madame Butterfly (Floros 1985, 244). Interessant ist jedoch, daß heterophone Strukturen schon in den Kindertotenliedern häufig auftreten (Bsp. 21 und 22). ${ }^{5}$ Prinzipiell ähnlich angelegt ist die folgende Passage aus dem Abschied, dem sechsten und letzten Satz des Lieds von der Erde (Bsp. 23). Die Kunst der kleinsten Abweichung scheint hier jedoch beinahe noch

4 Platon, auf dessen Nomoi der Begriff zurückgeht, rät ausdrücklich davon ab, junge Menschen mit dieser Form von Mehrstimmigkeit zu konfrontieren: »Mit Abweichungen aber und Variationen, in der Art, daß die begleitenden Töne der Leier sich nicht genau an die von dem Dichterkomponisten festgestellte Melodie halten, sowie mit jeder Gegensätzlichkeit von Stimme und Begleitung in Bezug auf die Zahl der Töne oder auf ihre langsamere oder schnellere Abfolge, oder auf ihre Höhe und Tiefe, und ebenso mit jeder selbständigen rhythmischen Variation der Begleitung durch die Töne der Leier - mit alledem müssen Unerwachsene verschont bleiben, die binnen drei Jahren in aller Eile die Elemente der Musik sich aneignen sollen. Denn das Gegensätzliche wirkt durch gegenseitige Störung nur hemmend auf den Unterricht ein, während man der Jugend die Wege zum Lernen möglichst ebnen muß (Platon 1988, 299). - Man kann den Lehrplänen der deutschen Musikhochschulen attestieren, daß sie Platons Ermahnung weitgehend beherzigen.

5 Adorno spricht in diesem Zusammenhang vom »unscharfen Unisono«, in welchem er ein »improvisatorisches Korrektiv der allzu ausgefegten Kunstlieder« sieht (Adorno 1971, 292). 


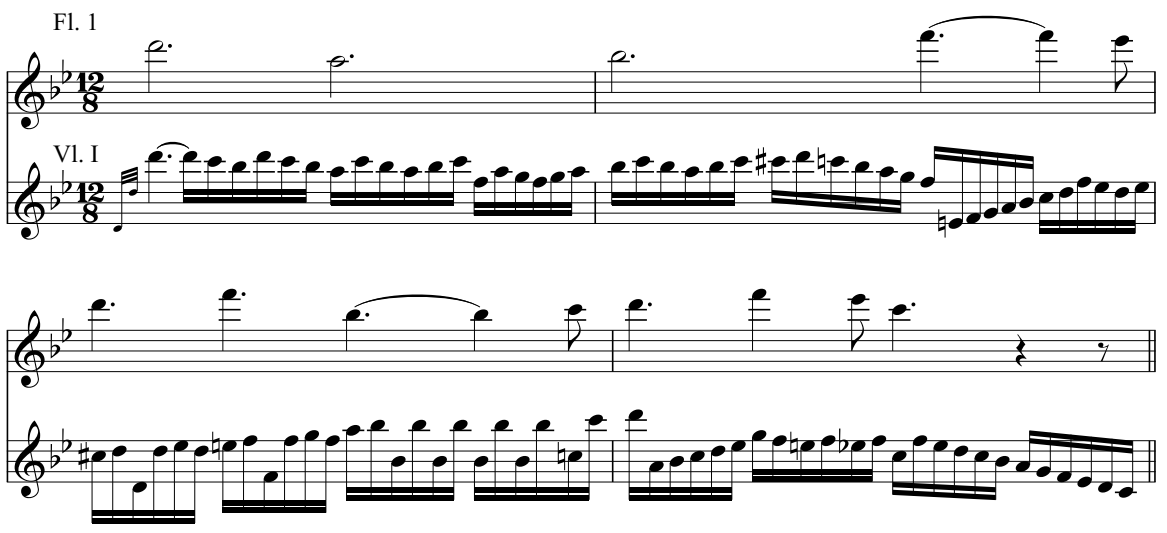

Beispiel 20: L. v. Beethoven: 9. Symphonie, III. Satz, T. 99-102

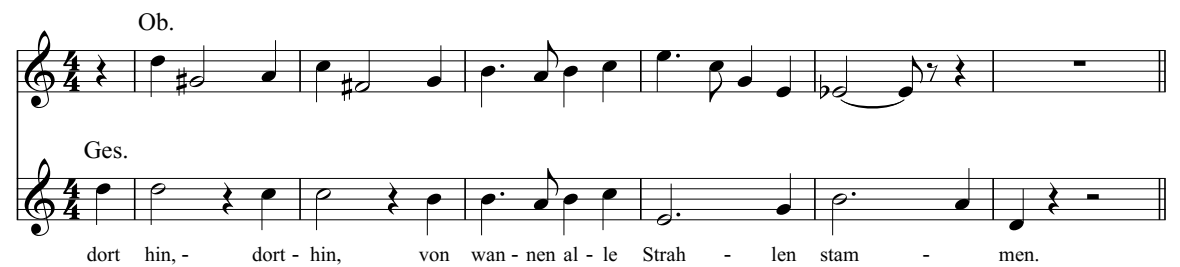

Beispiel 21: Gustav Mahler: Kindertotenlieder, II: Nun seh' ich wohl, warum so dunkle Flammen, T. 31-36

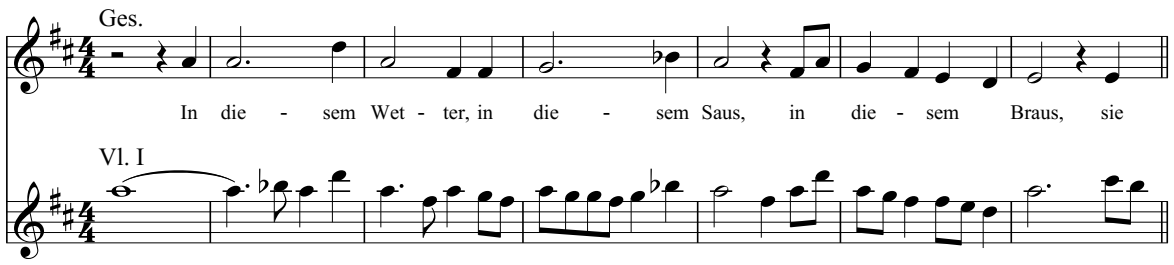

Beispiel 22: Gustav Mahler: Kindertotenlieder, V: In diesem Wetter, in diesem Braus, T. 100-106

weiter getrieben, so daß, trotz weitgehend identischen Melodieverlaufs zwischen Singstimme und dem Part der Violoncelli, beide Stimmen nur an zwei Punkten unmittelbar in ihrer Tonbewegung konvergieren, also tatsächlich Oktav- bzw. (wenn der Gesangspart von einem Bariton ausgeführt wird) Einklangsparallelen entstehen (Wechsel von T. 250 auf T. 251 und von diesem zu T. 252). 

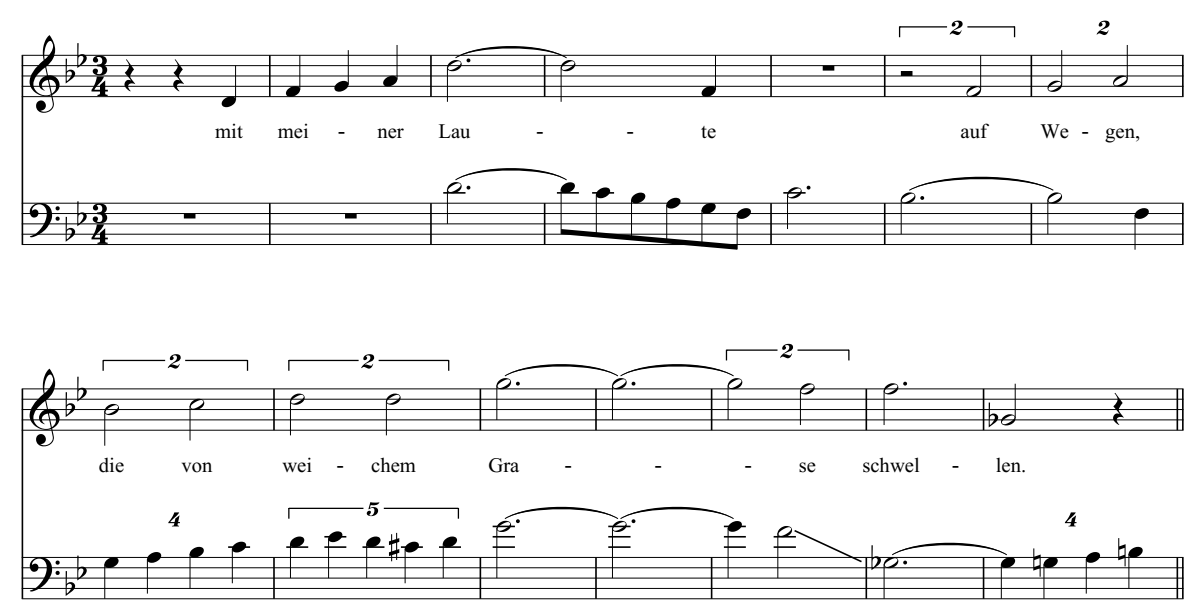

Beispiel 23: Gustav Mahler: Das Lied von der Erde, VI. Satz: Der Abschied, T. 243-256

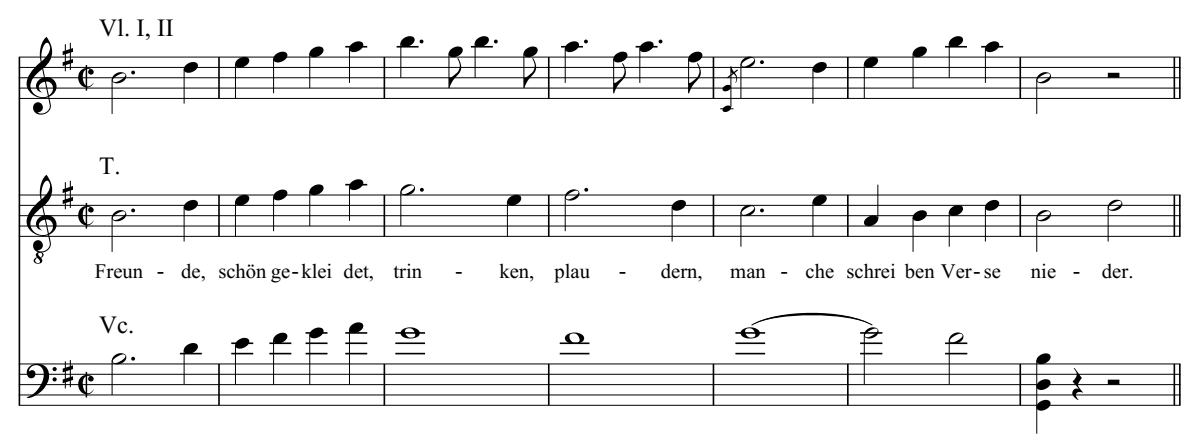

Beispiel 24: Gustav Mahler: Das Lied von der Erde, III. Satz: Von der Jugend, T. 41-47

Die beiden folgenden Beispiele spielen hingegen eher mit dem Wechsel von Gabelung und Zusammenführung mehrerer Stimmen auf sehr engem Raum. Geradezu didaktisch klar vollzieht sich in Bsp. 24 im zweitaktigen Rhythmus eine Entwicklung vom reinen Unisono über die Heterophonie (Simultanvariation) zur sechten` Polyphonie. Ständig neue Stimmkopplungen finden sich in Bsp. 25: Die durch die unterschiedlichen Mischungen bedingten Klangfarben sind einerseits in stetigem Wechsel begriffen, andererseits verhindert die weitgehende Kontinuität der einzelnen Partien eine Zersplitterung des Klangbildes in beziehungslose Einzelpartikel. ${ }^{6}$ Die vorübergehende Oktavierung der

6 Auffällig ist die Parallele von Mahlers Konzeption zu Richard Wagners orchestraler `Netztechnikı, die Diether de la Motte in Kontrapunkt. Ein Lese- und Arbeitsbuch eher skizziert als ausführlich darstellt. So schreibt de la Motte etwa zu einer Passage aus den Meistersingern von Nürnberg: "Ständig gehen mehrere Stimmen einen oder mehrere Schritte gemeinsam, trennen sich, um sich mit anderen 

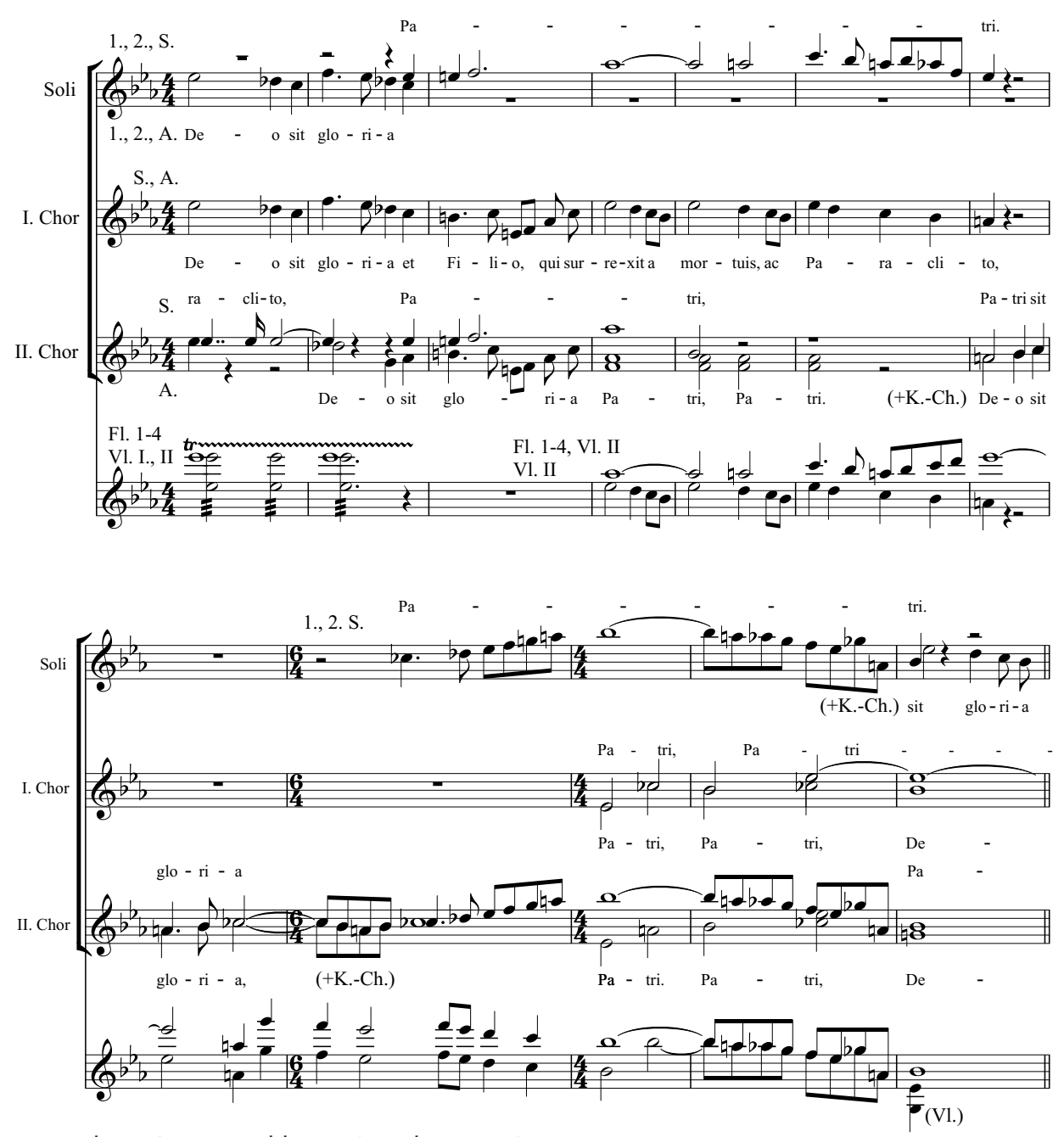

Beispiel 25: Gustav Mahler: 8. Symphonie, I. Satz, T. 532-543

Singstimme durch eine Solo-Violine in Bsp. 26 dient hingegen eher der motivischen Verknüpfung: Die Folge dreier absteigender Sekunden im Vokalpart (T. 1270-1273) ${ }^{7}$ wird

Stimmen kurzzeitig zum Unisono zu verbinden« (de la Motte 1982, 306); oder - bezogen auf das Verhältnis von Holzbläser- und Streichersatz im Lohengrin-Vorspiel: »Wichtig ist zu sehen, daß man sich nicht nur in gemeinsamen Tönen trifft, was bei kontrapunktischer Musik ja nichts Ungewöhnliches wäre, sondern daß gemeinsame Schritte ausgeführt werden, was als ıEinklangsparallelen aller kontrapunktischen Musik ein Greuel war« (ebd., 308). Ein detaillierter Vergleich zwischen Wagners und Mahlers Vorgehen wäre zweifellos von Interesse, kann im Rahmen dieser Arbeit jedoch nicht geleistet werden.

7 Strenggenommen nur die ersten beiden deszendenten Sekundschritte. 


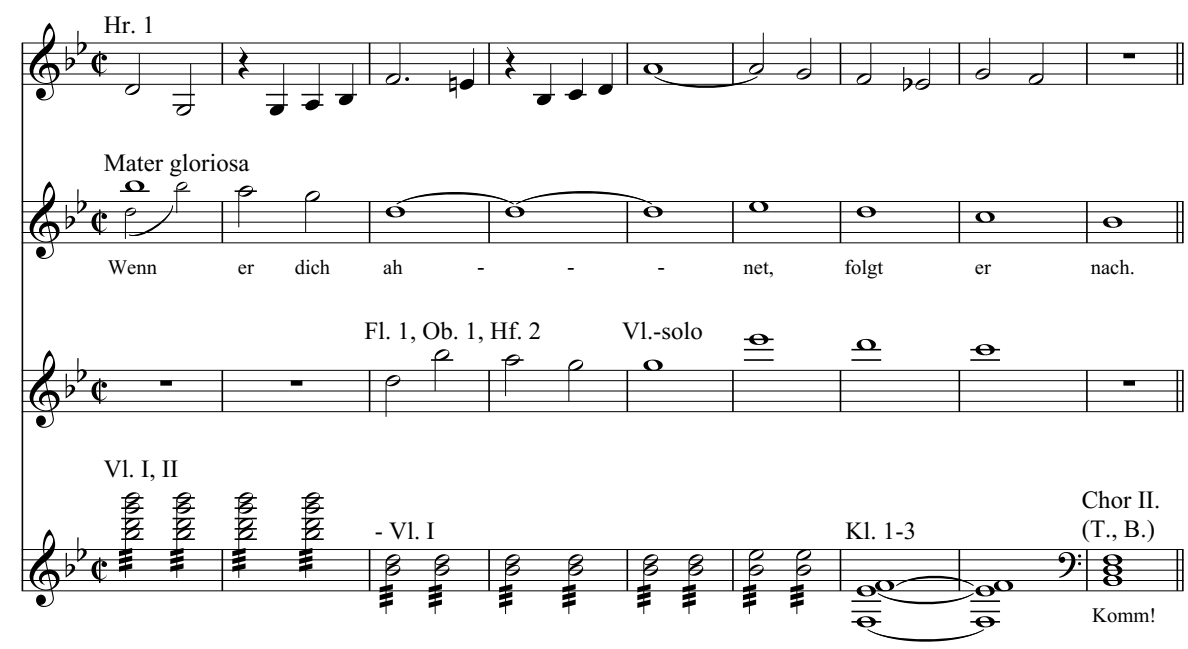

Beispiel 26: Gustav Mahler: 8. Symphonie, II. Satz, T. 1265-1273

gewissermaßen erst durch die Einblendung des viertönigen Motivs in der Solo-Violine (T. 1269-1272; vgl. Singstimme T. 1265/66 sowie Oboe/Harfe T. 1267/68) als Motivfragment erkannt - wenn man so möchte, eine Rudimentärform klingender Analyse, wie sie später etwa Anton Webern auf Bachs Ricercar a 6 aus dem Musikalischen Opfer angewandt hat.

Bei den meisten vorangegangenen Beispielen handelte es sich um musikalische Zusammenhänge, innerhalb derer die Prinzipien der traditionellen Polyphonie zurücktraten zugunsten heterophoner oder mixturähnlicher Strukturen. Am skritischsten sind Quint- und Oktavparallelen vielleicht dort, wo sie innerhalb eines polyphonen Idioms begegnen. Zumindest bei Quintparallelen ist dies im Werk Mahlers derart häufig der Fall, daß es verfehlt erschiene, bei jeder einzelnen Stelle nach einer besonderen `Begründung oder Motivierung zu fragen. Vielmehr erscheinen sie als quasi ıgleichberechtigte satztechnische Option, die zwar freilich weitaus seltener eingesetzt wird als etwa die Parallelführung von Terzen oder Sexten, aber dennoch als beinahe selbstverständlicher Bestandteil der musikalischen Sprache angesehen werden kann. So stellt sich eher die Frage nach Mahlers Verständnis von Polyphonie und Kontrapunkt überhaupt.

Klassische Polyphonie erscheint bei Mahler auf merkwürdige Weise gebrochen, verfremdet; dies mag nicht zuletzt von einer großzügigen Beimengung von Dorfmusikantentum herrühren, die die Prätention des Sublimen, Gelehrten von vorneherein desavouiert. Sehr deutlich wird dies in den folgenden Beispielen aus dem Finalsatz der 5. Symphonie (Bsp. 27 und 28). Gleich der erste Anlauf zur Imitationspolyphonie führt zu Quintparallelen zwischen `Dux` und `Comes` - welcher letztere, auffällig genug, den Dux in der Untersexte beantwortet -; die Begleitung der Fagotte und Celli mit ihrem tonikalen Orgelpunkt, ssatten Quinten im Zusammenklang und schlichtem Terzpendel in der Oberstimme verleiht der Passage eine deutlich pastorale Note (Bsp. 27). Noch drasti- 
Hr. 1, 3

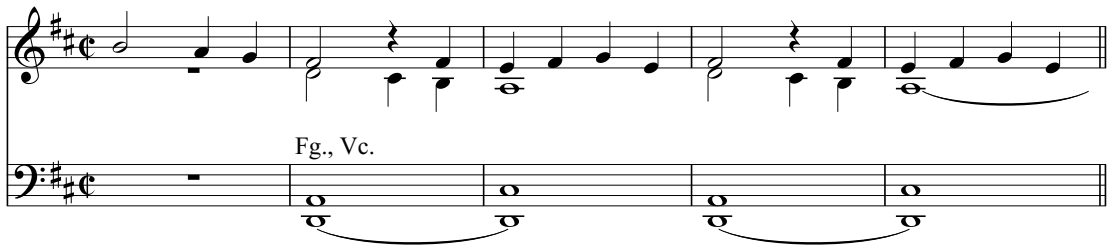

Beispiel 27: Gustav Mahler: 5. Symphonie, V. Satz, T. 24-28
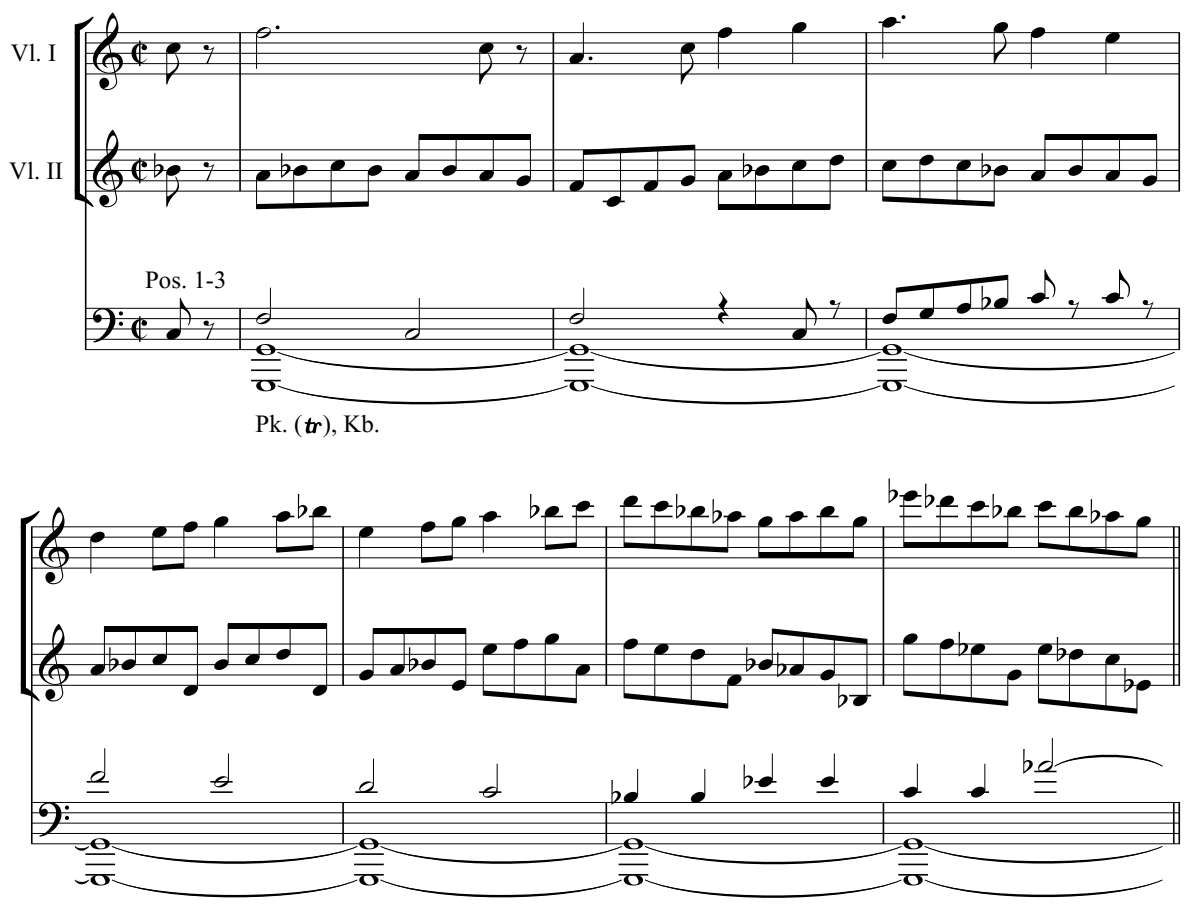

Beispiel 28: Gustav Mahler: 5. Symphonie, V. Satz, T. 558-564

scher scheinen die in Bsp. 28 zitierten Takte allem akademisch überlieferten Regelwerk zu spotten, versammeln sie doch auf engem Raum nicht weniger als sechs Quintparallelen: Das $>$ Allerweltsmäßige ${ }^{8}$ der Themen (in den 1. Violinen sowie in den Posaunen) findet seine Entsprechung in einem dilettantischen Tonsatz; selbst der Kontrapunkt der 2. Violinen ist bemerkenswert uninspiriert und erschöpft sich in tumber Betriebsamkeit. (Um hier nicht beim leeren Verdikt stehenzubleiben, sei auf die Sequenzenseligkeit bei-

8 Zu diesem Begriff vgl. Eggebrecht 1999, $100 f f$. 


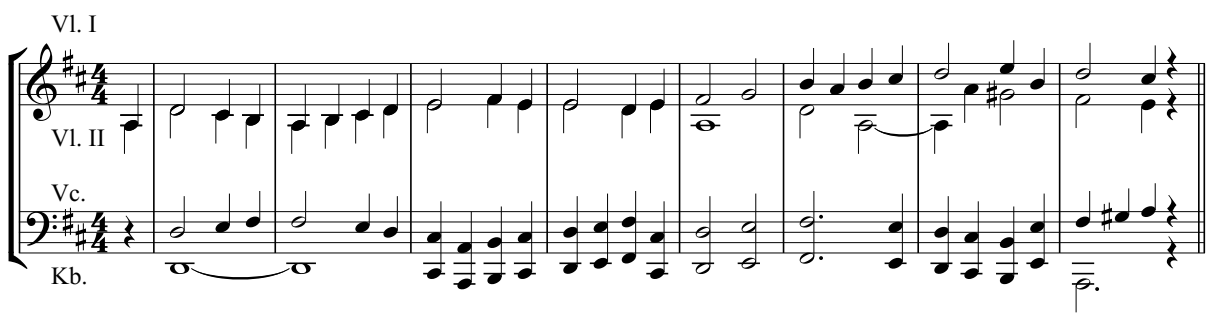

Beispiel 29: Gustav Mahler: 3. Symphonie, VI. Satz, T. 1-8

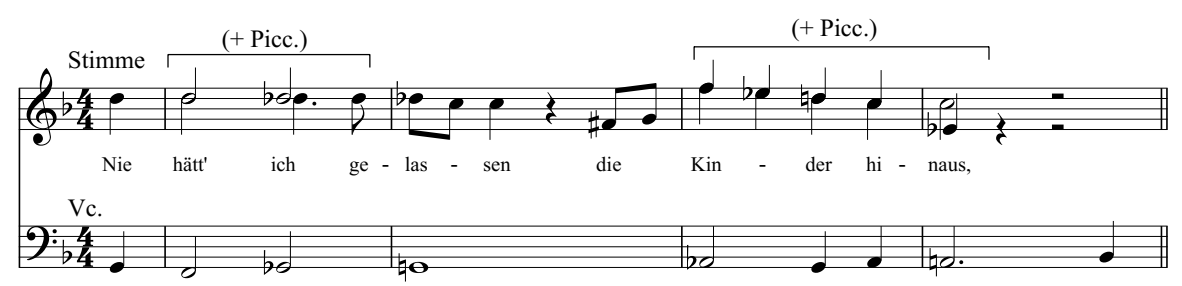

Beispiel 30: Gustav Mahler: Kindertotenlieder, V: In diesem Wetter, in diesem Braus, T. 54-57

1. A.

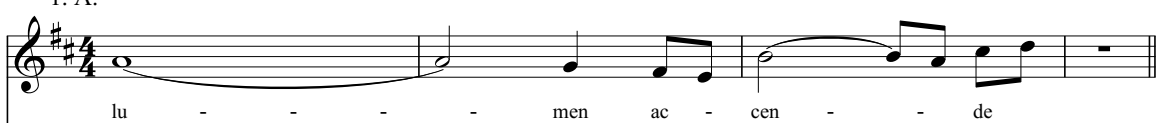

2. A., T.

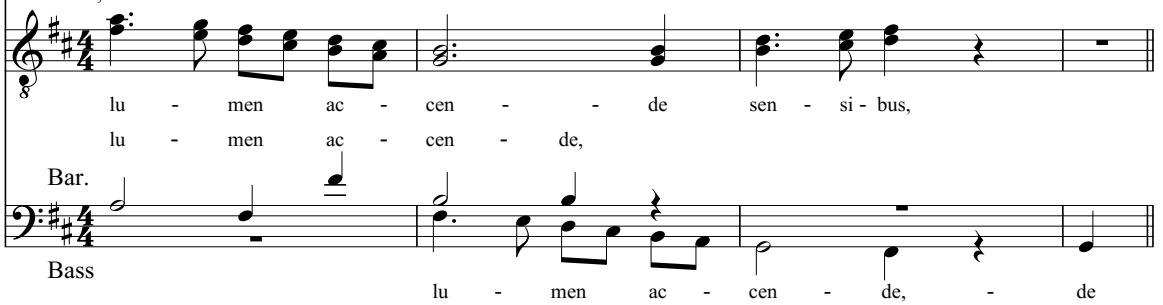

Beispiel 31: Gustav Mahler: 8. Symphonie, I Satz, T. 231-233

der Violinen ab T. 561 oder die übermäßige Abnutzung des Tons $C^{\prime \prime}$ in der 2. Violine [T. 558-561] hingewiesen).

Auffallend häufig begegnen Quintparallelen im Satz der Außenstimmen, wie in den folgenden Beispielen aus unterschiedlichen Schaffensperioden (Bsp. $29^{9}$, Bsp. 30, Bsp. 31).

9 Über den Beginn dieses Adagio-Satzes (Partituranweisung: »Langsam. Ruhevoll. Empfunden«) bemerkt Friedhelm Krummacher: „Und so regulär die Stimmführung erscheint, so wenig scheut sie vor Irregularitäten wie Quintparallelen zwischen den melodisch führenden Außenstimmen zurück (T. 2 [recte: T. 3] Violine I e'-fis', Cello A-H)« (Krummacher 1991, 149). 

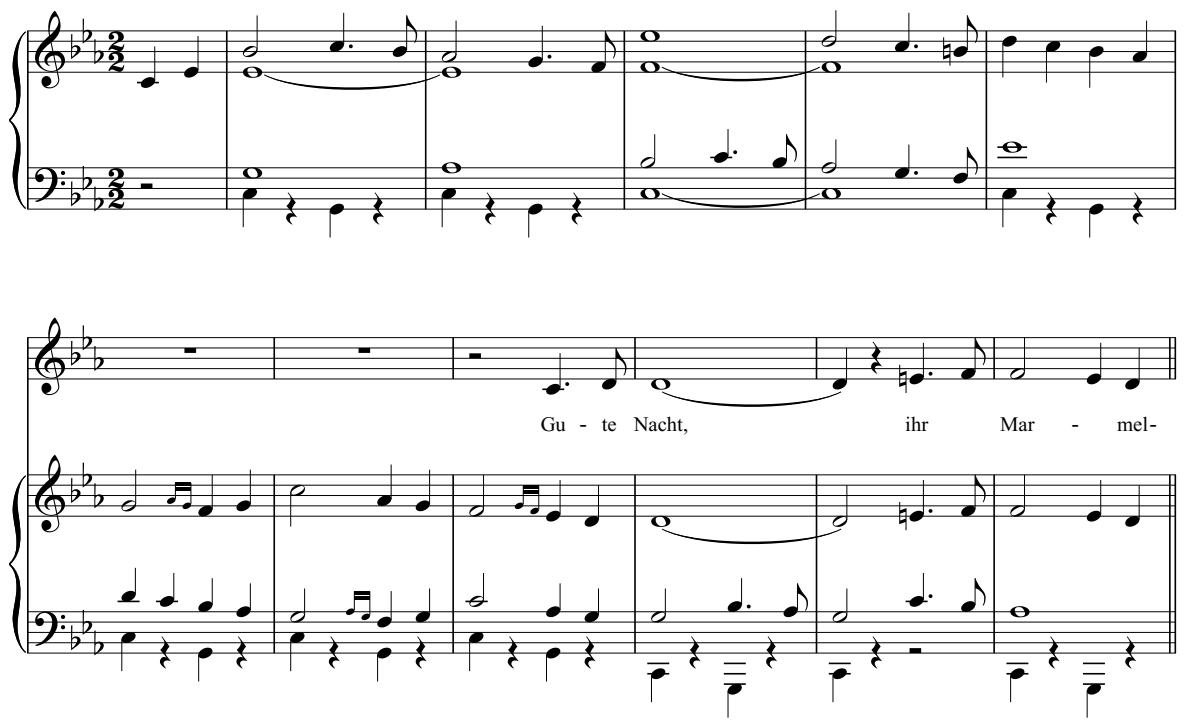

Beispiel 32: Gustav Mahler: Der Tamboursg'sell (Fassung für tiefe Stimme), T. 103-113

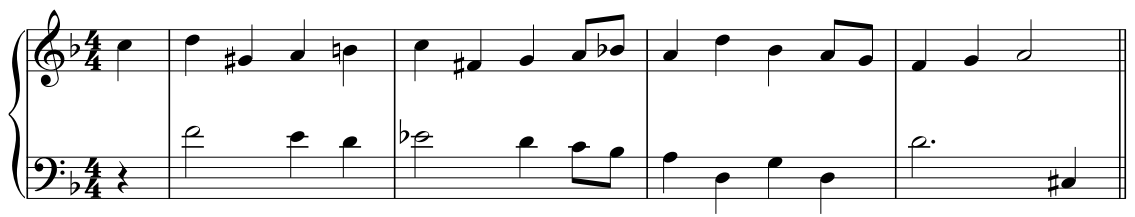

Beispiel 33: Gustav Mahler: Kindertotenlieder, I: Nun will die Sonn' so hell aufgeh'n, T. 1-4

Zum Abschluß drei Stellen, die aufgrund der geringeren Zahl der beteiligten Instrumente sowie infolge des ruhigen Grundtempos besonders gut durchhörbar sind (Bsp. 32). Dennoch gestehe ich, daß keine der in den folgenden Beispielen enthaltenen Quint- oder Oktavparallelen mir zuerst beim Hören aufgefallen ist - obwohl gerade die Oktavparallele im ersten der Kindertotenlieder (Bsp. 33), wenn man bewußt auf sie achtet, sich wie ein ıweißer Fleckı innerhalb der kurzen instrumentalen Einleitung ausnimmt. Mahlers verstärktes Interesse an Polyphonie - etwa ab der 5. Symphonie und der in ihrem zeitlichen Umfeld entstandenen Lieder (Sieben Lieder aus letzter Zeit; Kindertotenlieder) - wird in der Mahler-Literatur gelegentlich im Zusammenhang mit seiner intensiven Beschäftigung mit Johann Sebastian Bach gesehen. ${ }^{10}$ Natalie Bauer-Lechner gibt etwa folgende Sätze wieder: »[...] Unsagbar ist, was ich von Bach immer mehr und mehr lerne (freilich als Kind zu seinen Füßen sitzend): denn meine angeborene Art zu arbeiten ist Bachisch! Hätte ich nur Zeit, in diese höchste

10 So etwa bei Schreiber 1992, 146. 


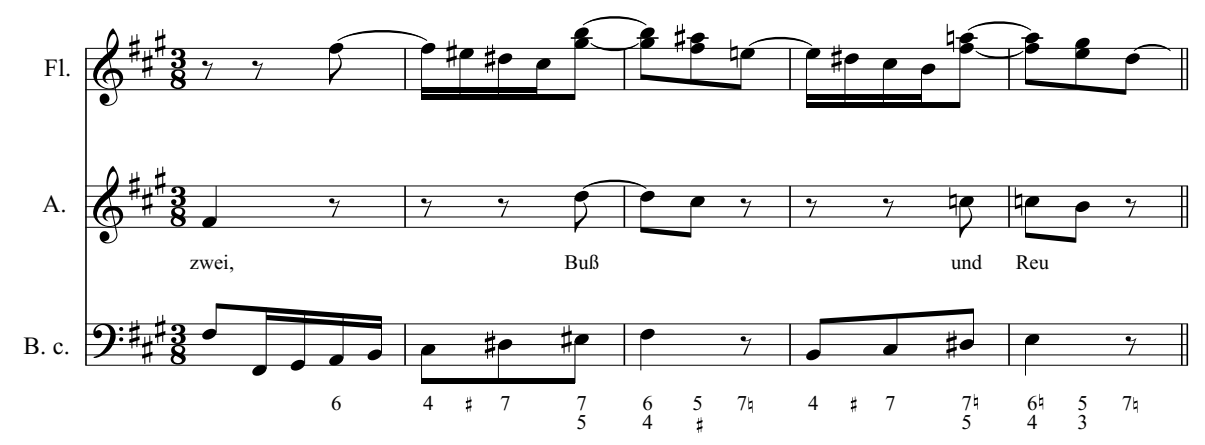

Beispiel 34: J. S. Bach: Matthäus-Passion, Alt-Arie Buß' und Reu', T. 49-52

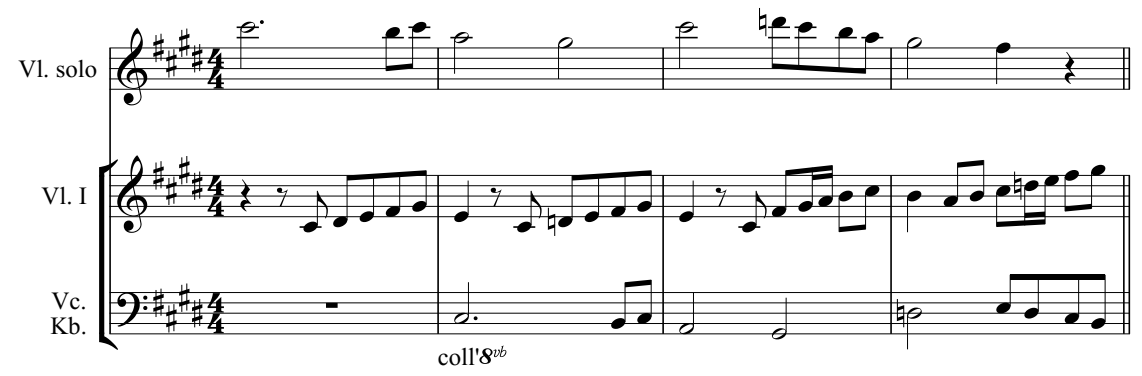

Beispiel 35: Gustav Mahler: 9. Symphonie, IV. Satz, T. 40-43

Schule mich ganz und gar zu versenken! Von welcher Bedeutung das wäre, kann ich selbst nicht ausdenken. Ihm aber seien meine späteren Tage, wenn ich endlich mir selbst gehöre, geweiht!« (Bauer-Lechner 1984, 189). Unter dem Datum 15. März 1899 berichtet sie von Mahlers Plänen einer Aufführung der Matthäus-Passion (ebd., 130), die jedoch nicht zustande kam. ${ }^{11}$ Erstaunlich ist, wie bachnahe und -fern zugleich der Beginn der Kindertotenlieder klingt: Die harmonische Progression der beiden Eingangstakte - wenn es legitim ist, Mahlers Zweistimmigkeit harmonisch zu deuten, was aber zumindest an dieser Stelle überaus naheliegt - könnte, als Sequenzierung eines Schmerzensmotivs, durchaus von einer Stelle wie der folgenden aus Bachs Matthäus-Passion (Bsp. 34) inspiriert sein. Gänzlich unbachisch hingegen sind die leeren Quarten (jeweils auf dem dritten Viertel), oder - anders betrachtet - die Repräsentation eines Dreiklangs nur durch dessen Grundton und Quinte. Die Quintparallele im zweiten Takt von Bsp. 35 schließlich ergibt sich aus einem nach den traditionellen Regeln sfehlerhaften doppelten Kontrapunkt.

11 Unter Verfechtern einer historischen Aufführungspraxis berüchtigt dürfte eine Bach-Bearbeitung sein, bei der Mahler einzelne Sätze aus den verschiedenen Orchester-Suiten zu einer eigenen Suite zusammenstellte. 
»Wie kaum für einen anderen gilt für Mahler, daß, was über den Standards ist, diesen zugleich nicht ganz genügt« (Adorno 1971, 167) - Adornos bewußt paradoxe Formulierung läßt sich auf Mahlers Verhältnis zum tradierten Tonsatz, wie mir scheint, durchaus schlüssig beziehen. Ob es freilich sinnvoller ist, Mahlers Satztechnik primär aus ihrer Relation (Überwindung, Negierung etc.) zu einem überlieferten Regelwerk oder aus ihrer Eigengesetzlichkeit, sozusagen einem ihr selbst immanenten Regelwerk, zu begreifen, soll hier nicht entschieden werden - wahrscheinlich erhellen sich beide Ansätze wechselseitig durch ihre Komplementarität.

Die in dieser Arbeit untersuchten Phänomene mögen sich, aus einem musikwissenschaftlichen Blickwinkel betrachtet, eher als Randphänomene von marginalem Interesse ausnehmen, für die Musiktheorie erscheinen sie mir insofern relevant, als satztechnische Praktiken des 19. - und beginnenden 20. - Jahrhunderts bislang in eher bescheidenem Ausmaß erforscht und beschrieben sind. Wenn etwa Werner Krützfeldt im KontrapunktArtikel der neuen MGG schreibt: „Die kompositorische Praxis des 19. Jh. außerhalb der Kirchenmusik benutzt im wesentlichen die überlieferten Stimmführungsregeln weiter, wobei durchgängig Kontrapunkt offensichtlich unverkrampft als harmonischen Grundsätzen untergeordnet interpretiert wird« (Krützfeld 1996, 621), so scheint zumindest die erste Aussage dieses Satzes mit der bei Mahler zu beobachtenden Praxis nicht ohne weiteres in Einklang zu bringen zu sein, auch wenn zugestanden sei, daß erstens die in dieser Arbeit zitierten Beispiele zu einem nicht unerheblichen Anteil aus dem ersten Jahrzehnt des 20. Jahrhunderts stammen und daß zweitens Krützfeldts Formulierung »im wesentlichen« Raum läßt für erhebliche Einschränkungen und Modifikationen. Doch gerade diese Unschärfe ist unbefriedigend: Wo beginnt einerseits das Wesen(tliche), Eigentliche, andererseits die bloße Oberfläche oder Erscheinungsform, wo hören beide auf? ${ }^{12}$ Der von Krützfeldt angesprochene Themenkomplex läßt sich wohl nur in einer Unmenge akribischer Detailarbeit erhellen, zumal `die « »kompositorische Praxis des 19. Jh." auseinanderfällt in ein babylonisches Gewirr unterschiedlichster, national, lokal, individuell oder auf sonstige Weise geprägter Praktiken.

Erfordert die Erforschung Mühe und Geduld, so erheischt die Deutung und Wertung in meinen Augen besondere Vorsicht: Gerne neigt man ja dazu, im Abweichenden das Fortschrittliche, ,Verdienstvolle zu sehen. Auch ich neige zu der These, daß Mahler durch die Lockerung allzu rigider Regeln der Musik neue Bereiche, gewiß neue Nuancen des Ausdrucks erschlossen hat. Dennoch gehört zu einem ıvollständigen ‘ahler-Bild - das es freilich ohnehin nicht gibt und nie geben wird - auch, daß Mahler selbst zumindest gewisse Regelwidrigkeiten wahrscheinlich als etwas durchaus Defizitäres angesehen hätte, wie die eingangs zitierten Sätze nahe genug legen. Aber auch an anderer Stelle finden sich aus Mahlers eigenem Munde Klagen über die Unzulänglichkeit seiner kontrapunktischen $>$ Routine $\triangleleft$ :

12 Vgl. hierzu etwa folgende kurze Passage aus dem Kapitel über Verschleierung des Stimmverlaufs bei Robert Schumann in de la Mottes Kontrapunkt: »Wer die Vernebelung als eine für die Essenz des Komponierten unwesentliche Zutat ansehen möchte, spiele die Takte nur einmal seindeutig‘[...]: Vom außerordentlichen Reiz der Stelle bleibt nichts, und auch ıvon Schumann wäre die Stelle nicht mehr!« (de la Motte 1981, 296). 
Jetzt begreife ich, daß Schubert, wie man erzählt, noch kurz vor seinem Ende Kontrapunkt studieren wollte. Er empfand, wie der ihm fehlte. Und ich kann ihm das nachfühlen, weil mir selbst dieses Können und ein richtiges, hundertfältiges Üben im Kontrapunkt aus der Lernzeit so abgeht. Da setzt nun an dessen Stelle bei mir allerdings der Intellekt ein, aber der Kräfteaufwand, der dazu erfordert wird, ist unverhältnismäßig groß (zit. nach Bauer-Lechner 1984, 159).

Um schließlich auch noch einmal auf Mahnkopfs ebenfalls zu Beginn dieser Arbeit wiedergegebene Charakterisierung von Mahlers Satzkunst zu kommen: Das Attribut des ,Unorthodoxen`, mehr noch das des »Winkelschiefen« scheint mir durchaus einen für die `Physiognomie von Mahlers Musik wesentlichen Punkt zu treffen, wohingegen die Rede von der «virtuose[n] Handhabung von Quintparallelen« in meinen Augen doch ein wenig fragwürdig ist. Zumindest bei den von mir zitierten Stellen aus Mahlers Symphonien und Liedern hat sich mir an keiner Stelle die Assoziation des Virtuosen aufgedrängt. So ziehe ich also am Ende meiner Arbeit über Quint- und Oktavparallelen bei Gustav Mahler das bescheidene Resümee: Es gibt sie.

\section{Literatur}

Adorno, Theodor W.: Mahler. Eine musikalische Physiognomik, in: Ders., Gesammelte Schriften 13: Die musikalischen Monographien, Frankfurt a. M. 1971.

Bauer-Lechner, Natalie: Erinnerungen an Gustav Mahler, hier zitiert nach der Ausgabe: Herbert Killian, Gustav Mahler in den Erinnerungen von Natalie Bauer-Lechner, mit Anmerkungen und Erklärungen von Knud Martner, Hamburg 1984.

Boulez, Pierre, Musikdenken heute 1 (=Darmstädter Beiträge zur Neuen Musik V), Mainz 1963.

Hans Heinrich Eggebrecht: Die Musik Gustav Mahlers, Wilhelmshaven 1999.

Floros, Constantin: Gustav Mahler. III: Die Symphonien, Wiesbaden 1985.

Krützfeld, Werner: Artikel »Kontrapunkt (19. Jahrhundert)«, in: Die Musik in Geschichte und Gegenwart. Allgemeine Enzyklopädie der Musik, begründet von Friedrich Blume, hg. von Ludwig Finscher, Bd. 5, Kassel 1996.

Krummacher, Friedhelm: Gustav Mahlers III. Symphonie. Welt im Widerbild, Kassel 1991. la Motte, Diether de: Kontrapunkt. Ein Lese- und Arbeitsbuch, Kassel 1981.

Mahnkopf, Claus-Steffen: »Mahlers Gnosis«, in: Musik-Konzepte 91: Gustav Mahler - Der unbekannte Bekannte, hg. von Heinz-Klaus Metzger und Rainer Riehn, München 1996, 34-45.

Mann, Thomas: Doktor Faustus. Das Leben des deutschen Tonsetzers Adrian Leverkühn erzählt von einem Freunde, Stockholm 1947, hier zitiert nach der Ausgabe: Frankfurt a. M. 1990.

Platon: Gesetze. Siebentes Buch. Übers. von Otto Apelt, Hamburg 1988.

Schreiber, Wolfgang: Gustav Mahler, Reinbek bei Hamburg ${ }^{16} 1992$. 